\title{
ORGANOLEPTIC AND CHEMICAL PROPERTIES OF COCONUT AND SESAME OILS AND THEIR BLENDS
}

\author{
L.L.W.C. Yalegama, C. Jayasekara, S.A.R. Upali ${ }^{1}$, M.J. \\ Abeygunawardhana ${ }^{1}$
}

Coconut Research Institute, Lunuwila ${ }^{1}$ University of Peradeniya, Peradeniya

\begin{abstract}
The chemical properties of six blends of coconut and sesame oils, and the organoleptic qualities of the blends and foods prepared with them were studied. The blends prepared for testing were: (1) 100\% coconut oil; (2) 90 \% coconut and $10 \%$ sesame; (3) $75 \%$ coconut and $25 \%$ sesame; (4) $50 \%$ coconut and 50 \% sesame; (5) $25 \%$ coconut and $75 \%$ sesame; and (6) 100 $\%$ sesame oil.

In sensory evaluations, on a Five-point Hedonic scale, all the oil blends were found to be acceptable for deep frying of fish and oil cakes, and shallow frying of noodles. Oil blends 1, 2 and 3, in descending order, received high sensory scores (4.2-3.8) for taste of deep fried fish; blends 6, 4 and 5 scored less (3.0 points each), but were acceptable. Blend 3 received the highest score of 4.3 for taste of both oil cakes and noodles; other blends ranged from 3.6-3.9.

Colour and smell of oil blends 6, 5 and 4 scored only 2.2-2.5 and were not acceptable. Blends with $75 \%$ or more of coconut oil scoring 4.2-3.8 for colour and 3.7-3.3 for smell, were acceptable. All the oil blends stored well up to five months without exceeding the prescribed SLS standard for FFA values for edible coconut (white oil) and sesame oils. Blends 1, 2 and 3, containing less than $50 \%$ sesame oil, can be stored for 6 months. After 6 months FFA levels showed an increasing trend. Iodine value did not change significantly but Peroxide values increased steadily during storage. Oil blends with higher proportions of coconut oil had relatively low peroxide values. Fatty acid composition of coconut oil can be altered by blending with sesame oil to increase the unsaturated fatty acid content of coconut oil without affecting its organoleptic qualities.
\end{abstract}

Key words: sesame oil, saturated fatty acids, monounsaturated fatty acids, polyunsaturated fatty acids, oil blends 


\section{INTRODUCTION}

Fats and oils are of major importance in human nutrition. They provide energy, helps the body absorb fat soluble vitamins, A, D, E and K, and supply essential fatty acids. Essential fatty acids are fatty acids that cannot be synthesized in the human body from precursors and therefore have to be provided in the diet. Fats and oils improve the texture and palatability, and also add characteristic flavours to food preparations.

Coconut being an extensively cultivated plantation crop in Sri Lanka, its oil is the most popular cooking oil in the country. Coconut oil is widely used for both shallow and deep frying, in domestic and commercial scale food preparation. Coconut oil imparts desirable flavour, texture and taste to food. Sesame oil is also used as a cooking oil in Sri Lanka. Sesame is cultivated and processed on a small scale in the drier parts of Sri Lanka and a limited quantity of the unrefined oil is available in the market. Sesame oil is traditionally used by the Tamil community in Sri Lanka and it is their preferred cooking oil. Most others find its smell and taste less than agreeable.

Coconut oil is categorized as a lauric oil, and contains a high percentage of lauric acid, a medium chain fatty acid, and very small amounts of essential fatty acids. Approximately $60 \%$ of the fatty acids in coconut oil are short and medium chain saturated fatty acids. Short and medium chain fatty acids are easily digestible. All the other vegetable oils are rich in essential fatty acids and contain high amounts of long chain fatty acids. Nutritionists advice the intake of an oil that contains saturated, monounsaturated and polyunsaturated fatty acids in the ratio 1:1:1 (Bockisch, 1998). A vegetable oil with a balanced fatty acid composition is rare or non-existent and brings to the fore the importance of blended vegetable oils. In many countries edible oil is marketed as a blend of two or three oils (Murthi et al, 1987). For example, Lokdhara is a blend of $67 \%$ palm olein and 33\% ground nut oil (Semwal and Arya, 2001).

Sesame oil is a highly unsaturated oil, rich in essential fatty acids (Bockisch, 1998). Thus, blending coconut and sesame oils to produce a cooking oil with a desirable composition of fatty acids, including essential fatty acids, and with acceptable organoleptic properties appears to be a feasible proposition. Therefore a study was undertaken to prepare different blends of coconut oil and sesame oil and evaluate the organoleptic and chemical properties of the oil blends, and also foods prepared with the blends. As coconut oil is by far the most popular cooking oil in Sri Lanka the blends would target coconut oil users. 


\section{MATERIAL AND METHODS}

Coconut oil used in the study was obtained from the Coconut Research Institute and the sesame oil was procured from a sesame oil mill in Kekirawa area in Anuradhapura District. Both oils were unrefined. Four oil blends with different proportions of coconut and sesame oils were prepared for testing along with the straight oils as references. The oils were mixed for 20 minutes with a magnetic stirrer and stored in glass bottles at ambient conditions. The coconut oil to sesame oil ratios of the blends and their colour are given in Table 1.

Table 1. Ratio of coconut oil to sesame oil in the blends and their colour

\begin{tabular}{ccl}
\hline Blend No. & Ratio Coconut : Sesame & Colour \\
\hline 01 & $100: 0$ & Light yellow \\
02 & $90: 10$ & Light yellow \\
03 & $75: 25$ & Yellow \\
04 & $50: 50$ & Brownish yellow \\
05 & $30: 70$ & Brownish yellow \\
06 & $0: 100$ & Light brown \\
\hline
\end{tabular}

Chemical properties of oil blends such as Free fatty acid (FFA) content, Peroxide value and iodine value were measured over six months at monthly intervals as per AOCS (1990). The blends were analysed for their fatty acid composition using Gas Chromatography (AOCS, 1990).

Organoleptic qualities of the oil blends were evaluated in four sensory evaluation tests: one to evaluate the colour and smell of the oil blends, and three sensory tests to evaluate deep fried and shallow fried foods prepared with the oil blends. The evaluations were made by 21 semi-trained panelists. Five point hedonic scale, with a score of 5 for 'like very much', 4 for "like slightly", 3 for "neither like nor dislike" 2 for "dislike slightly and 1 for 'dislike very much' was used to evaluate the degree of liking, for a particular quality attribute. A score of 3.0 or higher denotes that the attribute is generally acceptable.

Randomized complete block design was used to evaluate chemical properties and the Friedman Ranking test was used for sensory evaluations. Analysis of variance $(\mathrm{P}<0.05)$ was performed on the sensory evaluation scores using the MINITAB statistical package (MINITAB Inc., State Collage, PA). 


\section{RESULTS AND DISCUSSION}

\section{Organoleptic qualities of the oil blends}

The sensory evaluation carried out for the degree of liking of colour and smell of the oil blends showed significant differences $(\mathrm{P}<0.05)$ between them. Blends 1, 2 and 3 with $100 \%$ coconut oil, $90 \%$ coconut \& $10 \%$ sesame, and $75 \%$ coconut \& $25 \%$ sesame, had scores greater than 3 for both colour and smell indicating their acceptance by the consumer (Table 2). Blends 4, 5 and 6 which contained 50\%-100\% sesame oil scored less than 3, and were not acceptable. The 100\% coconut oil was light yellow and 100\% sesame oil was light brown in colour (Table 1). Blend No.2 (90\% coconut \& 10\% sesame) had the highest score for colour (4.2 out of 5.0).

Table 2. Degree of liking of the colour and smell of oil blends, scored on a Five-point hedonic scale.

\begin{tabular}{|c|c|c|c|}
\hline \multirow{2}{*}{ Blend No. } & Blend composition & \multicolumn{2}{|c|}{ Estimated medians } \\
\cline { 3 - 4 } & $100 \%$ coconut & 3.8 & Colour \\
\hline 01 & $\begin{array}{c}\text { Smell } \\
\text { sesame }\end{array}$ & 4.2 & 3.7 \\
\hline 02 & $\begin{array}{c}75 \% \text { coconut }: 25 \% \\
\text { sesame }\end{array}$ & 3.8 & 3.3 \\
\hline 03 & $\begin{array}{c}50 \% \text { coconut }: 50 \% \\
\text { sesame }\end{array}$ & 2.5 & 2.3 \\
\hline 04 & $\begin{array}{c}30 \% \text { coconut }: 70 \% \\
\text { sesame }\end{array}$ & 2.5 & 2.2 \\
\hline 06 & $100 \%$ sesame & 2.2 & 2.2 \\
\hline
\end{tabular}

\section{Organoleptic qualities of foods prepared with the oil blends}

\section{Fried fish}

Fresh fish was deep fried in the different oil blends and evaluated for organoleptic qualities of appearance, crispness and taste on the five-point hedonic scale. All six oil blends scored 4.0 out of 5.0 for appearance, and approximately 4.0 for crispness, with blend No. 2 (90\% coconut \& 10\% sesame) and blend No. 5 (30\% coconut \& 70\% sesame) receiving the highest and lowest scores respectively (Table 3). Fried fish prepared with 100\% coconut oil and blend No. 2 (90\% coconut \& 10\% sesame) received 4.2 and 4.0 respectively for taste; blend No.3 (75\% coconut \& $25 \%$ sesame) scored 
3.8 and the other blends scored 3.0. In the five-point hedonic scale a score of 3.0 is regarded as acceptable. Therefore all six blends tested were acceptable for deep frying of fish.

\section{Kevum (Oil cake)}

The oil blends were tested for their suitability for preparing a traditional food like oil cake (Kevum). Oil cakes prepared with different blends were evaluated for their smell and taste. All six oil blends scored 3.8 out of 5.0 for the smell of Kevum (Table 3) but the scores for taste differed. Kevum prepared with blend No. 3 (75\% coconut \& 25\% sesame) was the most preferred scoring 4.3 for taste. All the other blends received scores of 3.6 to 3.9. Thus all six oil blends are acceptable for the preparation of Kevum.

Table 3. Degree of liking for the sensory attributes of foods prepared with oil blends, scored on a five-point hedonic scale.

\begin{tabular}{|c|c|c|c|c|c|c|c|}
\hline \multirow{2}{*}{$\begin{array}{c}\text { Blend } \\
\text { No. }\end{array}$} & \multicolumn{7}{|c|}{ Estimated medians } \\
\cline { 2 - 8 } & Appearance & Crispness & Taste & Smell & Taste & Smell & Taste \\
\hline 01 & 4.0 & 3.9 & 4.2 & 3.8 & 3.9 & 3.8 & 3.6 \\
\hline 02 & 4.0 & 4.1 & 4.0 & 3.8 & 3.7 & 3.8 & 3.7 \\
\hline 03 & 4.0 & 3.9 & 3.8 & 3.8 & 4.3 & 3.8 & 4.3 \\
\hline 04 & 4.0 & 3.9 & 3.0 & 3.8 & 3.6 & 3.8 & 3.9 \\
\hline 05 & 4.0 & 3.8 & 3.0 & 3.8 & 3.6 & 3.8 & 3.6 \\
\hline 06 & 4.0 & 3.9 & 3.0 & 3.8 & 3.6 & 3.8 & 3.6 \\
\hline
\end{tabular}

\section{Noodles}

The oil blends were tested for their suitability for shallow frying by preparing a popular food like noodles. Noodles prepared with different oil blends were evaluated for their smell and taste. All six oil blends scored 3.8 out of 5.0 for the smell of noodles. Blend No. 3 (75\% coconut \& 25\% sesame) was the most preferred for taste, scoring 4.3 out of 5 . All the other blends were acceptable and received scores of 3.6 to 3.9. Accordingly, while all the blends tested are acceptable, blend No. 3, 75\% coconut \& 25\% sesame is the best blend for preparation of shallow fried foods like noodles. It is interesting to note that for shallow frying the oil blends were superior to the straight oils, which received the lowest scores. 


\section{Chemical properties of the oil blends}

\section{Free fatty acid content}

Free fatty acid (FFA) content is an indicator of the keeping quality of a cooking oil. The SLS standard for the FFA content of edible coconut (white oil) and sesame oil is less than $1 \%$, and $3 \%$ respectively. The FFA content of the oil blends recorded, at monthly intervals, over the six-month storage period are presented in Fig. 1. The initial FFA values conform to the SLS standard. The initial FFA values of the blends were raised with the addition of sesame oil. The FFA content of $100 \%$ coconut oil increased from $0.032 \%$ up to $0.930 \%$ and $100 \%$ sesame oil increased from $2.46 \%$ up to $3.7 \%$, during the six month storage period.

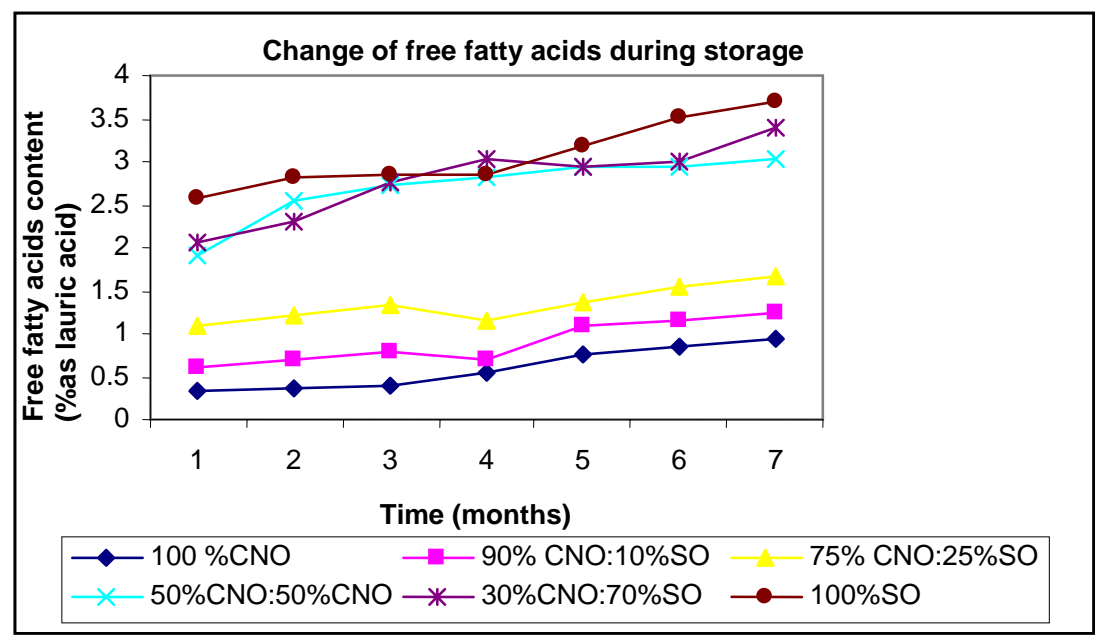

Figure 1. Effect of storage on the free fatty acid content of oil blends

\section{Peroxide value}

Initial peroxide values of $100 \%$ coconut oil and $100 \%$ sesame oil was 3.08 meqv/kg and $1.68 \mathrm{meqv} / \mathrm{kg}$ respectively. The peroxide values of oil blends recorded, at monthly intervals, over the six-month storage period are presented in Fig. 2. Peroxide values of all the oil blends increased rapidly during storage. This could be ascribed to the oils being unrefined. Relatively low peroxide values were recorded in the blends containing high percentages of coconut oil (100\% to $75 \%$ coconut oil). Peroxide values of these blends appeared to reach an equilibrium state, while the others showed a steady increase during the storage period. 


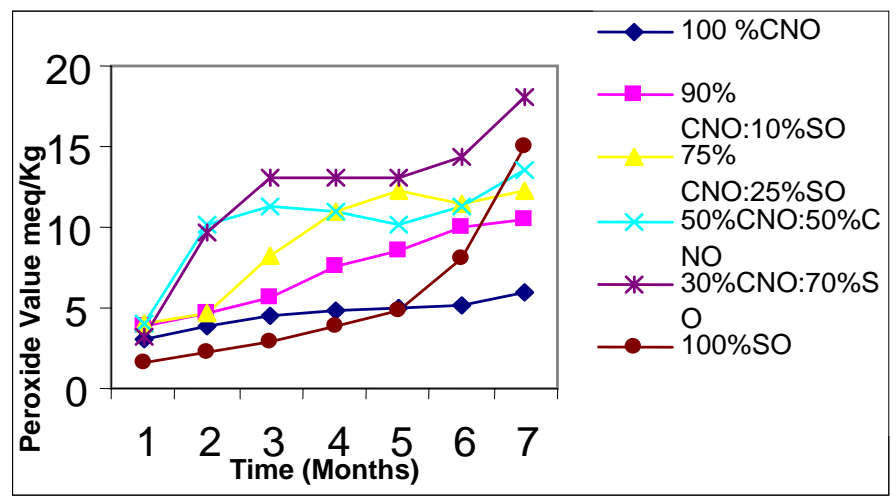

Figure 2: Effect of storage on the Peroxide value of the oil blends

\section{Iodine value}

The initial iodine values of coconut oil and sesame were 7.3 and 103.3 respectively, which indicates that sesame oil is highly unsaturated compared to coconut oil. As to be expected, the iodine value of the blends increased as the proportion of sesame oil increased. Iodine values of the pure oils and their blends did not change significantly during storage.

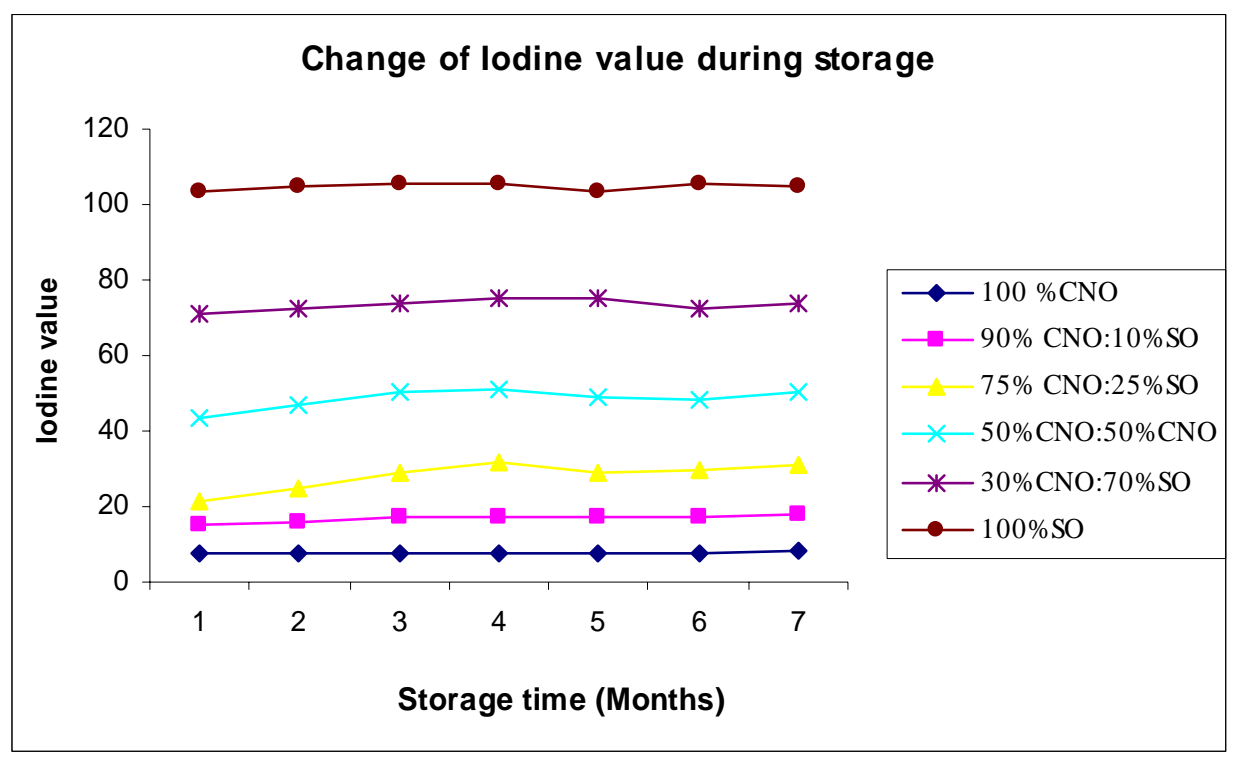

Figure 3: Effect of storage on the Iodine value of oil blends 


\section{Fatty acid composition of oil blends}

Percentage content of fatty acids, and of the saturated, monounsaturated and polyunsaturated fatty acid types (SFA, MUFA and PUFA), in the oil blends, are presented in Tables 3 and 4 respectively. The major constituent of coconut oil was Lauric acid (48.96\%). Oleic acid (41.83\%) and Linoleic acid (43.62\%) were the major constituents of sesame oil. Increasing the sesame oil content in the blend decreases Lauric acid content and increases Oleic and Linoleic acid content. For instance, Blend No. 3 with $75 \%$ coconut oil and $25 \%$ sesame oil, has 41.03 \% Lauric acid , $11.04 \%$ Oleic acid and $11.10 \%$ Linoleic acid, and a SFA:MUFA:PUFA ratio of 78: 11:11. There was a better balance of fatty acids in Blend No. 5 (30\% coconut oil and 70\% sesame oil) with 21.27\% Lauric acid, 25.69\% Oleic acid and $27.34 \%$ Linoleic acid, and a SFA:MUFA:PUFA ratio of 47: 26:27.

Table 3: Percentage content of fatty acids in the oil blends

\begin{tabular}{|l|c|c|c|c|c|c|}
\hline \multirow{2}{*}{ Fatty acid } & \multicolumn{7}{|c|}{ Blend No. } \\
\cline { 2 - 7 } & 1 & 2 & 3 & 4 & 5 & 6 \\
\hline Caproic (C6:0) & 2.11 & 2 & & & & \\
\hline Caprilic (C8:0) & 8.65 & 7.79 & 7.45 & 4.36 & 3.06 & \\
\hline Capric (C10:0) & 5.52 & 4.97 & 4.96 & 2.75 & 2.27 & \\
\hline Lauric (C12:0) & 48.96 & 44.06 & 41.03 & 24.85 & 21.27 & \\
\hline Myristic (C14:0) & 17.4 & 14.76 & 14.23 & 9.2 & 7.68 & \\
\hline Palmitic (C16:0) & 7.14 & 7.82 & 7.37 & 9.65 & 8.86 & 9.36 \\
\hline Stearic (18:0) & 2.32 & 2.61 & 2.82 & 4 & 3.83 & 5.19 \\
\hline Oleic (C18:1) & 6 & 9.28 & 11.04 & 22.43 & 25.69 & 41.83 \\
\hline Linoleic (C18:2) & 1.9 & 6.71 & 11.1 & 22.76 & 27.34 & 43.62 \\
\hline
\end{tabular}

1 - $100 \%$ coconut oil, 2 - $90 \%$ coconut oil and $10 \%$ sesame oil, 3 - $75 \%$ coconut oil and $25 \%$ sesame oil, 4 - $50 \%$ coconut oil and $50 \%$ sesame oil, 5 - $30 \%$ coconut oil and $70 \%$ sesame oil, 6 - $100 \%$ sesame oil 
Table 4. Percentage content of SFA, MUFA and PUFA in the oil blends

\begin{tabular}{|c|c|c|c|c|c|c|}
\hline \multirow{2}{*}{ Fatty acid } & \multicolumn{7}{|c|}{ Blend No. } \\
\cline { 2 - 7 } & 1 & 2 & 3 & 4 & 5 & 6 \\
\hline SFA & 92 & 84 & 78 & 55 & 47 & 15 \\
\hline MUFA & 6 & 9 & 11 & 22 & 26 & 42 \\
\hline PUFA & 2 & 7 & 11 & 23 & 27 & 44 \\
\hline
\end{tabular}

SFA - Saturated fatty acids; MUFA - Monounsaturated fatty acid; PUFA Polyunsaturated fatty acids

1 - $100 \%$ coconut oil, 2 - $90 \%$ coconut oil and $10 \%$ sesame oil, 3 - $75 \%$ coconut oil and $25 \%$ sesame oil, 4 - $50 \%$ coconut oil and $50 \%$ sesame oil, 5 - $30 \%$ coconut oil and $70 \%$ sesame oil, 6 - $100 \%$ sesame oil

The deficiency in essential fatty acids is the main disadvantage of coconut oil. Pure coconut oil (Blend No.1) contains only 6\% MUFA and 1.9\% PUFA while pure sesame oil (Blend No. 6) contains 41.83\% MUFA and 43.62\% PUFA. MUFA and PUFA contain essential fatty acids that are necessary for growth. These essential fatty acids are not synthesized in the human body and have to be supplied by way of food. If a blend of sesame and coconut oils, such as Blend No. 5, is used in food preparation it would provide a good source of essential fatty acids. In this regard it is noteworthy that in sensory evaluations Blend No 5 proved to be acceptable for preparation of oil cakes, deep frying of fish and shallow frying of noodles.

\section{CONCLUSIONS}

Blends of coconut and sesame oil stored well for approximately 6 months. The chemical parameters, FFA, iodine value and peroxide value increased during storage. However, the FFA content of the blends did not exceed the limits specified for the pure oils. Fatty acid composition of the highly saturated coconut oil (92.1\% SFA) can be altered by blending with sesame oil to increase the unsaturated fatty acid content, without affecting its organoleptic qualities. A blend of 30\% coconut oil and $70 \%$ sesame oil had a SFA:MUFA:PUFA ratio of 47:26:27 and contained $21.27 \%$ Lauric acid, 25.69\% Oleic acid and 27.34\% Linoleic acid. The blended oils were suitable for preparing a wide range of foods. They can be used for deep frying of fish, preparation of oil cake and stir-frying noodles. In sensory evaulations the organoleptic qualities of food prepared with oil blends were found to be acceptable. 


\section{REFERENCES}

Bockisch, M. (1998). Importance of fats in Fats and oils hand book. AOCS press, Champaign, Illinois.

Semwal, A. D., Arya, S.S. (2001) Studies on the stability of some edible oils and their blends during storage. J. Food Sci. Technol., 38 (5): 515518.

Murthi, T.N., Sharma, M., Devadhara, V.D., Chatterjee, S., Chakraborty, B.K. (1987). Storage stability of edible oils and their blends. J Food Sci. Technol 24: 84-87.

AOCS (1990). Official methods and recommended practices of the American Oil Chemist's Society, $4^{\text {th }}$ Edn. Champaign, Illinois 